\title{
Basic Principles and Applied GxP Regulations for ELISA Analytical Method Validation of Drugs and Biologics in FDA Driven Environment
}

\author{
Article by Diana Karagiozova \\ Clinical Research, Texila American University \\ E-mail: Diana.karagyozova@gmail.com
}

\begin{abstract}
The Food and Drug Administration (FDA) is a federal agency of the United States that is responsible for protecting the health of the public by ensuring the security, efficacy, and safety of both the veterinary and human drugs. The agency is also responsible for ensuring the safety of the food supply, radioactive materials, and cosmetics in the United States. The FDA has a statutory duty of ensuring that manufacturers of various products, including pharmaceuticals, comply with their respective established Good Manufacturer Practice (GMP).

ELISA (enzyme-linked Immunosorbent analysis) is an important bioanalytical assay format that pharmaceutical companies particularly use to validate in order to achieve a robust method suitable for the purpose of development of drugs and biologics in FDA regulated environment.

The analytical laboratories carrying out all toxicology or pharmacology as well as other pre-clinical studies for the purposes of making regulatory submissions are expected to comply with FDA's Good Laboratory Practices (GLPS) and sound quality assurance principles throughout the validation of appropriate ELISA methods and/or testing process. As long as pharmaceutical companies evaluate analytical methods before and during regular use, they are not under any statutory obligation to comply with the FDA guidelines on method validation, including bioanalytical methods such as ELISA methods.
\end{abstract}

Keywords: FDA, GXP, Impurity ELISA, sandwich ELISA, validation. Basic Principles and Applied GxP Regulations for ELISA Analytical Method Validation of Drugs and Biologics in FDA Driven Environment.

\section{Regulations}

The Food and Drug Administration (FDA) is a federal agency of the of the United States Department of Health and Human Services (HHS) that is responsible for protecting the health of the public by ensuring the security, efficacy, and safety of both the veterinary and human drugs (Food and Drug Administration, 2017a). According to the Food and Drug Administration (2017a), the FDA is also responsible for ensuring the safety of the food supply, radioactive materials, and cosmetics in the United States. In brief, the FDA has a statutory duty of ensuring that manufacturers of various products, including pharmaceuticals, comply with their respective established Good Manufacturer Practice (GMP). In particular, the FDA has put in place the necessary mechanisms that are aimed at monitoring how drug manufacturers comply with its Current Good Manufacturing Practice (CGMP) regulations so as to guarantee the quality of drug products (Food and Drug Administration, 2017b). The Food and Drug Administration (2017c) explains that the CGMPs provide for systems that guarantee "proper design, monitoring, and control of manufacturing processes and facilities." The significance of manufactures adhering to the CGMP regulations is that it assures the purity, quality, strength, and identity of drug products by requiring that medication manufacturers adequately control their manufacturing operations (Food and Drug Administration, 2017c). Specifically, this control of the manufacturing operations includes the establishment of strong quality management systems, acquisition of suitable quality raw materials, and an establishment of robust operating procedures, identifying and investing any deviations in product quality, and maintaining reliable testing laboratories (Food and Drug Administration, 2017c). The Food and Drug Administration (2017c) argues that if pharmaceutical companies adequately put into practice the CGMP regulations, it would help them to prevent incidences of deviations, mix-ups, errors, failures, and contamination. Consequently, this would guarantee that drug products from these pharmaceutical companies meet their quality standards. 
Subsequently, in an effort to bolster the existing formal system of controls at pharmaceutical companies through the CGMP regulations, the Food and Drug Administration (2011) has established general principles and practices for the validation process. These general principles and practices, according to the Food and Drug Administration (2011), are suitable elements that pharmaceutical companies should use in process validation for the manufacture of animal and human biological and drug products, including the active pharmaceutical ingredients (APIs). In other words, the general principles and practices that the FDA has established the validation process incorporate the approaches and principles that all the manufacturers of medications can use to validate the manufacturing process. The Food and Drug Administration (2011) defines process validation as "the collection and evaluation of data, from the process design stage through commercial production, which establishes scientific evidence that a process is capable of consistently delivering quality product" (p. 4). The process entails a series of activities that take place over a product's lifecycle and process. FDA guide on the general principles and processes for process validation describes the validation activities in three key stages: process design, process qualification, and continued process verification. It is important to point out that there has been a recent inquest as to whether the CGMPs require three successful process validation batches before a new API or a finished drug product is released for distribution (Food and Drug Administration, 2017d). In response, the FDA asserts that neither its policy nor the CGMP regulations specify a minimum number of batches to validate a manufacturing process (Food and Drug Administration, 2017d). Moreover, the existing FDA guidance on APIs also does not stipulate the number of batches for any process validation. Nonetheless, the FDA recommends that manufactures should expand their testing based on the established validation protocol so as to provide an added guarantee that a batch satisfies all the appropriate and established criteria before API is used in the final drug product (Food and Drug Administration, 2017d).

Incidentally, the FDA has established a guideline that pharmaceutical companies should use to validate bioanalytical procedures such as high-pressure liquid chromatography (LC), gas chromatography (GC), combined LC and GC mass spectrometric (MS) procedures such as LC-MSMS, GC-MS-MS, and LC-MS carried out for the quantitative determination of metabolites and/or drugs in biological matrices such as urine, serum, or blood (Food and Drug Administration, 2001). Food and Drug Administration (2001) adds that this bioanalytical method validation guidance for the pharmaceutical industry also applies to other bioanalytical procedures such as microbiological and immunological procedures, and to other biological matrices, for example, skin and tissue samples. Moreover, the guidance could be used in enzyme-linked immunosorbent assay (ELISA) tests.

\section{ELISA}

In order to fully understand the methodological approaches that the proposed research intends to use in evaluating the validation method for each of the two types of ELISA, it is imperative to first provide a brief background on sandwich (indirect method) ELISA and impurity ELISA. The sandwich ELISA, according to Adams and Moss (2007) and Ferenčík (2012), is a robust and sensitive method that measures the concentration of an antigen in an unknown sample. Ferenčik (2012) elucidates that the antigen of interest in sandwich ELISA is usually quantified between two layers of antibodies: the detection and capture antibody. The author adds that the detection and capture antibody must bind to the antigen's non-overlapping epitopes. It is important to note that either the affinity-purified polyclonal or monoclonal antibodies can be utilized as the capture and detection antibodies (Adams \& Moss, 2007). However, this is dependent on the cost, the dynamic range, and sensitivity of the final assay. Yamazaki et al. (2010) observe that sandwich procedures can sometimes be difficult to optimize and consequently, it is recommended that a tested and matching pair of antibodies should be used. The significance of using a tested matching pair of antibodies is that it guarantees that the antibodies are detecting various epitopes on the target protein in order not to interfere with another antibody binding.

The impurity ELISA format, on the other hand, quantify the residual impurities present in an assay. The residual impurities usually include Insulin, CHO HCP, rProtein A, and beta Glucan. In particular, the impurity ELISA methods used commercially available kits (particularly for early phase molecules) such as the spiking sample in matrix to evaluate the level of endogenous impurities in a sample. Different kits have different set-up and requirements for reagent handling. 


\section{Current industry practices}

The bioanalytical methods in support of pharmacokinetic studies are considered to be among the most challenging types of analytical methods to develop and validate. Essentially, this means that ELISAs make up the more challenging bioanalytical methods to develop and validate. It is important to note that the selectivity and sensitivity of bioanalytical methods are fundamental to the success of both the pre-clinical and clinical pharmacology studies (Swartz \& Krull, 2003). Swartz and Krull (2003) contend that just like any other analytical method, it is a requirement for the performance characteristics of a particular bioanalytical method to be shown by recorded laboratory data, to be reliable and reproducible to its intended purpose of use.

Subsequently, the Food and Drug Administration (2001) acknowledges that in a regulated laboratory such as that of a pharmaceutical company, the process of validating any type of analytical method does not start with the method in question. The software and any instrument that is used first in the validation process must be validated or qualified based on the current SOPs, and any data generated has to be maintained in compliance with the established FDA electronic and signature rules (Food and Drug Administration, 2001; Swartz \& Krull, 2003). Swartz and Krull (2003) assert that it is only after the stage has been set that the validating process of a bioanalytical method can be separated into three parts: preparation of the reference standard, development of the bioanalytical method and the establishment of the procedure for the validated assay, and the application of the validated method in the regular drug analyses. After performing a full validation study on a bioanalytical method, laboratories often conduct partial validation to modify the existing validated analytical methods and cross-validation to compare two or more methods or to transfer methods. Nonetheless, the bottom line of the full and partial validation procedures is that using laboratory data to record to a particular method is appropriate and reliable for its intended use never changes. Fundamentally, this makes up the basic principle of method validation, regardless of the type of analytical method that is under scrutiny (Swartz \& Krull, 2003, Shah, 2007; Galvao et al., 2008; Mattocks et al., 2010).

\section{GAPS Identified}

The FDA regulations such as the GLP, GMP, and other quality standards require that analytical methods should be evaluated before and during regular use. Moreover, there are no particular regulations on method validation. Nevertheless, the FDA, other government agencies, and industry task forces have developed guidelines for validating methods. Therefore, this means that as long as pharmaceutical companies evaluate analytical methods before and during regular use, they are not under any statutory obligation to comply with the FDA guidelines on method validation, including ELISA. It is incumbent upon these companies to either consider using the recommended FDA guidelines on method validation or to develop and implement a method validation that guarantees the security, efficacy, and safety of the drug product as provided under the FDA guidelines. Hence, pharmaceutical companies can, in fact, develop and adopt an ELISA test validation method that best suits their drug product

The FDA has only developed guidelines that can help pharmaceutical companies in developing and implementing a validation method for an analytic process such as the ELISA tests. Consequently, various pharmaceutical companies have developed different method validation processes for their analytical methods. Since the majority of the pharmaceutical companies have differences in handling entire validation process, the emphasis should be on some basic concepts of validation in regular indirect ELISA assays as well as impurity methods for ELISA. For this purpose, a validation report should be represented, and a draft method has to be in place. All Validation activities must be in accordance and compliant with the International Conference on Harmonization (ICH) Q2 (R1) Guidelines for the Validation of Analytical.

\section{Remedy and conclusion}

The researchers shall place significant emphasis on examining the method validation steps of two types of ELISA: the binding ELISA format (sandwich ELISA) and impurity ELISA format.

Also, the FDA shall publish a detailed guidance on how to handle a validation/co-validation activities for both formats and/or establish clear criteria for a robust method validation process. 
In order to ensure that the findings of the proposed research are reliable, the researcher shall conduct both the internal and external validity of the proposed study design. Fundamentally, the study's internal validity shall entail the researcher addressing all the relevant parameters, and appropriate ELISA principles are implemented during the execution of the experiments. For example, the researcher shall ensure that the set parameters such as the incubation time, reagent dilution, coating concentration, and the standard curve parameters are performed as laid out in the established practices. In a nutshell, the researcher shall take all the necessary steps to protect the integrity of the study design. Incidentally, the external validity of the study design shall encompass co-validation of the proposed design with those that have been used in other laboratories. In other words, the researcher shall compare the findings drawn from the proposed research with those generated by similar studies from other laboratories. It is important to point out that the proposed research shall only compare its findings to those that have been produced using the ELISA test and not any other bioanalytical method. The coefficient variation $(\mathrm{CV})$ that shall be used during co-validation shall be set below $20 \%$ for both laboratories and thereby conform to the traditional $\mathrm{CV}$ in most studies.

Furthermore, data from the experiment shall be subjected to statistical analysis so that the findings can be interpreted and appropriate inferences made from the research (Nachar, 2008; De Winter \& Dodou, 2010; MacFarland \& Yates, 2016). In particular, the researcher shall employ the Mann-Whitney $\mathrm{U}$ test to statistically analyze the collected data. The Mann-Whitney U test is described as the nonparametric alternative test to the traditional independent sample t-test (Nachar, 2008; De Winter \& Dodou, (2010), Fay \& Proschan, 2010). As a non-parametric test, the Mann-Whitney U test is used to compare two sample means that are drawn from the same population, and is used to determine whether two sample means are equal or otherwise (Black, 2009; Milenović, 2011). It is critical to point that in practice, the Mann-Whitney $U$ test is used in statistical analysis when the assumptions of the t-test are not satisfied or the data in question is ordinal. Since the proposed research shall involve the use of samples with unknown antibody concentrations and some with impurities, it is easy to predict that the data is highly likely to be ordinal. Therefore, the Mann-Whitney $\mathrm{U}$ test would be ideal in the proposed research.

Moreover, a factorial design with center points for Binding ELISA format to set up appropriate parameters shall be used. In particular, the factorial design shall involve the selection of factors for the experiment, for example, the capture antibody concentration, detection antibody concentration, plate wash, reagent incubation time, specific dilutions of antibody, substrate incubation time. The importance of setting the parameters for the study is because these parameters shall be evaluated later during analytical method validation. The particular analytical method validation parameters that the proposed research shall seek to evaluate in so far as the sandwich ELISA is concerned to include specificity, accuracy, linearity, repeatability, Intermediate precision, Limit of Detection and QL (quantitation limit) and statistical analysis using JMP software analysis.

Depending on the needs of the user, the user must identify and select the method of ELISA analysis. The choice of the method analysis must be one that solves the analytical problem and shows its suitability for the intended purpose. Fundamentally, the aim of this proposed research is to evaluate the validation processes in in ELISA methods of analysis. An important part of the validation processes is to study and estimate all the parameters that are needed for good performance.

\section{References}

[1].Adams, M. R., \& Moss, M. (2007). Food microbiology. Royal society of chemistry.

[2].Black, K. (2009). Business statistics: Contemporary decision making. John Wiley \& Sons

[3].Ferenčík, M. (2012). Handbook of immunochemistry. Springer Science \& Business Media.

[4].Food and Drug Administration (2001). Guidance for Industry: Bioanalytical Method Validation. Fda.gov. Retrieved 18 September 2017, from www.fda.gov/downloads/Drugs/Guidance/ucm070107.pdf

[5].Food and Drug Administration (2011). Guidance for Industry Process Validation: General Principles and Practices. Fda.gov. Retrieved 18 September 2017, from www.fda.gov/downloads/drugs/guidances/ucm070336.pdf.

[6].Food and Drug Administration (2017a). What We Do. Fda.gov. Retrieved 18 September 2017, from www.fda.gov/AboutFDA/WhatWeDo/default.htm 
[7].Food and Drug Administration (2017b). Drug Applications and Current Good Manufacturing Practice (CGMP) Regulations. Fda.gov. Retrieved 18 September 2017, from www.fda.gov/drugs/developmentapprovalprocess/manufacturing/ucm090016.htm.

[8].Food and Drug Administration (2017c). Facts about the Current Good Manufacturing Practices (CGMPs). Fda.gov. Retrieved 18 September 2017, from

www.fda.gov/drugs/developmentapprovalprocess/manufacturing/ucm169105.htm.

[9].Food and Drug Administration (2017d). Questions and Answers on Current Good Manufacturing PracticesProduction and Process Controls. Fda.gov. Retrieved 18 September 2017, from www.fda.gov/Drugs/GuidanceComplianceRegulatoryInformation/Guidances/ucm124782.htm\#5.

[10]. Milenović, Ž. (2011). Application of Mann-Whitney U test in research of professional training of primary school teachers. Metodički obzori, 6(11), 73-79.

[11]. Nachar, N. (2008). The Mann-Whitney U: A test for assessing whether two independent samples come from the same distribution. Tutorials in Quantitative Methods for Psychology, 4(1), 13-20.

[12]. Nachar, N. (2008). The Mann-Whitney U: A test for assessing whether two independent samples come from the same distribution. Tutorials in Quantitative Methods for Psychology, 4(1), 13-20.

[13]. Swartz, M., \& Krull, I. (2003). Validation of Bioanalytical Methods-Highlights of FDA's Guidance. LCGC North Amer, 21, 136-142.

[14]. Yamazaki, Y., Imura, A., Urakawa, I., Shimada, T., Murakami, J., Aono, Y., ... \& Okamoto, N. (2010). Establishment of sandwich ELISA for soluble alpha-Klotho measurement: age-dependent change of soluble alpha-Klotho levels in healthy subjects. Biochemical and biophysical research communications, 398(3), 513-518. 